\title{
The Role of Adiposity in Cardiometabolic Traits: A Mendelian Randomization Analysis
}

Tove Fall ${ }^{1,29}$, Sara Hägg ${ }^{1,29}$, Reedik Mägi, ${ }^{3,49}$, Alexander Ploner², Krista Fischer ${ }^{4}$, Momoko Horikoshi, ${ }^{3,5}$, Antti-Pekka Sarin ${ }^{6}$, Gudmar Thorleifsson ${ }^{7}$, Claes Ladenvall ${ }^{8}$, Mart Kals ${ }^{4}$, Maris Kuningas ${ }^{9}$, Harmen H. M. Draisma ${ }^{10,11}$, Janina S. Ried ${ }^{12}$, Natalie R. van Zuydam ${ }^{13}$, Ville Huikari ${ }^{14}$, Massimo Mangino ${ }^{15}$, Emily Sonestedt ${ }^{16}$, Beben Benyamin ${ }^{17,18}$, Christopher P. Nelson ${ }^{19,20}$, Natalia V. Rivera ${ }^{21,22,23}$, Kati Kristiansson ${ }^{24}$, Huei-yi Shen ${ }^{6,25}$, Aki S. Havulinna ${ }^{24}$, Abbas Dehghan ${ }^{9,26}$, Louise A. Donnelly ${ }^{13}$, Marika Kaakinen ${ }^{14,27}$, MarjaLiisa Nuotio ${ }^{24}$, Neil Robertson ${ }^{3,5}$, Renée F. A. G. de Bruijn 9,28, M. Arfan Ikram 9,28,29, Najaf Amin ${ }^{23}$, Anthony J. Balmforth ${ }^{30}$, Peter S. Braund ${ }^{19}$, Alexander S. F. Doney ${ }^{13}$, Angela Döring ${ }^{31,32}$, Paul Elliott $^{33}$, Tõnu Esko ${ }^{4}$, Oscar H. Franco ${ }^{9,26}$, Solveig Gretarsdottir ${ }^{7}$, Anna-Liisa Hartikainen ${ }^{34}$, Kauko Heikkilä ${ }^{35}$, Karl-Heinz Herzig ${ }^{27,36,37}$, Hilma Holm ${ }^{7}$, Jouke Jan Hottenga ${ }^{10,11}$, Elina Hyppönen ${ }^{38}$, Thomas Illig ${ }^{39,40}$, Aaron Isaacs ${ }^{23}$, Bo Isomaa ${ }^{41,42}$, Lennart C. Karssen ${ }^{23}$, Johannes Kettunen $^{6,24}$, Wolfgang Koenig ${ }^{43}$, Kari Kuulasmaa ${ }^{24}$, Tiina Laatikainen ${ }^{24}$, Jaana Laitinen ${ }^{44}$, Cecilia Lindgren ${ }^{3}$, Valeriya Lyssenko ${ }^{8,45}$, Esa Läärä ${ }^{46}$, Nigel W. Rayner ${ }^{3,5,47}$, Satu Männistö ${ }^{24}$, Anneli Pouta ${ }^{34,48}$, Wolfgang Rathmann ${ }^{49}$, Fernando Rivadeneira ${ }^{26,50}$, Aimo Ruokonen ${ }^{51}$, Markku J. Savolainen ${ }^{27,52}$, Eric J. G. Sijbrands ${ }^{50}$, Kerrin S. Small ${ }^{15}$, Jan H. Smit ${ }^{11,53,54}$, Valgerdur Steinthorsdottir ${ }^{7}$, Ann-Christine Syvänen ${ }^{55}$, Anja Taanila ${ }^{14}$, Martin D. Tobin ${ }^{56}$, Andre G. Uitterlinden ${ }^{50}$, Sara M. Willems ${ }^{23}$, Gonneke Willemsen ${ }^{10,11}$, Jacqueline Witteman ${ }^{9,26}$, Markus Perola ${ }^{4,24}$, Alun Evans ${ }^{57}$, Jean Ferrières ${ }^{58}$, Jarmo Virtamo ${ }^{24}$, Frank Kee ${ }^{59}$, DavidAlexandre Tregouet ${ }^{60}$, Dominique Arveiler ${ }^{61}$, Philippe Amouyel ${ }^{62}$, Marco M. Ferrario ${ }^{63}$, Paolo Brambilla ${ }^{64}$, Alistair S. Hall ${ }^{30}$, Andrew C. Heath ${ }^{65}$, Pamela A. F. Madden ${ }^{65}$, Nicholas G. Martin ${ }^{17}$, Grant W. Montgomery ${ }^{17}$, John B. Whitfield ${ }^{17}$, Antti Jula ${ }^{66}$, Paul Knekt ${ }^{67}$, Ben Oostra ${ }^{68}$, Cornelia M. van Duijn ${ }^{23,69,70}$, Brenda W. J. H. Penninx ${ }^{11,54}$, George Davey Smith ${ }^{71}$, Jaakko Kaprio ${ }^{6,35,72}$, Nilesh J. Samani ${ }^{19,20}$, Christian Gieger ${ }^{12}$, Annette Peters ${ }^{32,73}$, H.Erich Wichmann ${ }^{31,74,75}$, Dorret I. Boomsma ${ }^{10,11,53}$, Eco J. C. de Geus ${ }^{10,11,53}$, TiinaMaija Tuomi ${ }^{42,76}$, Chris Power $^{38}$, Christopher J. Hammond ${ }^{15}$, Tim D. Spector ${ }^{15}$, Lars Lind ${ }^{77}$, Marju Orho-Melander ${ }^{8}$, Colin Neil Alexander Palmer ${ }^{13}$, Andrew D. Morris ${ }^{13}$, Leif Groop ${ }^{6,8}$, Marjo-Riitta Järvelin ${ }^{14,27,48,78}$, Veikko Salomaa ${ }^{24}$, Erkki Vartiainen ${ }^{79}$, Albert Hofman ${ }^{9,26}$, Samuli Ripatti ${ }^{6,24,47}$, Andres Metspalu4, Unnur Thorsteinsdottir ${ }^{7,80}$, Kari Stefansson 7,80 , Nancy L. Pedersen ${ }^{2 \uparrow}$, Mark I. McCarthy ${ }^{3,5,81 \%}$, Erik Ingelsson ${ }^{1,2,39_{*}}$, Inga Prokopenko ${ }^{3,5,829_{*}}$, for the European Network for Genetic and Genomic Epidemiology (ENGAGE) consortium

1 Molecular Epidemiology and Science for Life Laboratory, Department of Medical Sciences, Uppsala University, Uppsala, Sweden, 2 Department of Medical Epidemiology and Biostatistics, Karolinska Institutet, Stockholm, Sweden, $\mathbf{3}$ Wellcome Trust Centre for Human Genetics, University of Oxford, Oxford, United Kingdom, 4 Estonian Genome Center, University of Tartu, Tartu, Estonia, 5 Oxford Centre for Diabetes, Endocrinology and Metabolism, University of Oxford, Oxford, United Kingdom, 6 Institute for Molecular Medicine Finland, University of Helsinki, Helsinki, Finland, 7 deCODE Genetics, Reykjavik, Iceland, 8 Department of Clinical Sciences, Diabetes and Endocrinology, Lund University Diabetes Centre, Lund University and Skåne University Hospital, Malmö, Sweden, 9 Department of Epidemiology, Erasmus Medical Center, Rotterdam, The Netherlands, 10 Department of Biological Psychology, VU University Amsterdam, Amsterdam, The Netherlands, 11 The EMGO Institute for Health and Care Research, Amsterdam, The Netherlands, 12 Institute of Genetic Epidemiology, Helmholtz Zentrum München-German Research Center for Environmental Health, Neuherberg, Germany, 13 Medical Research Institute, Ninewells Hospital and Medical School, University of Dundee, Dundee, United Kingdom, 14 Institute of Health Sciences, University of Oulu, Oulu, Finland, 15 Department of Twin Research and Genetic Epidemiology, King's College London, United Kingdom, 16 Diabetes and Cardiovascular Diseases Genetic Epidemiology Research Unit, Department of Clinical Sciences, Skåne University Hospital, Lund University, Malmö, Sweden, 17 Queensland Institute of Medical Research, Herston, Australia, 18 Queensland Brain Institute, University of Queensland, St Lucia, Australia, 19 Department of Cardiovascular Sciences, University of Leicester, Leicester, United Kingdom, 20 National Institute for Health Research, Leicester Cardiovascular Biomedical Research Unit, Glenfield Hospital, Leicester, United Kingdom, 21 IRCSS Multimedica, Milan, Italy, 22 Institute of Genetics and Biomedical Research, Consiglio Nazionale delle Ricerche, Milan, Italy, 23 Department of Genetic Epidemiology, Erasmus Medical Center, Rotterdam, The Netherlands, 24 Department of Chronic Disease Prevention, National Institute for Health and Welfare, Helsinki, Finland, $\mathbf{2 5}$ Public Health Genomics Unit, National Institute for Health and Welfare, Helsinki, Finland, 26 Netherlands Consortium for Healthy Ageing, Netherlands Genomics Initiative, Leiden, The Netherlands, 27 Biocenter Oulu, University of Oulu, Oulu, Finland, 28 Department of Neurology, Erasmus Medical Center, Rotterdam, The Netherlands, 29 Department of Radiology, Erasmus Medical Center, Rotterdam, The Netherlands, 30 Division of Epidemiology, Leeds Institute of Genetics, Health and Therapeutics, School of Medicine, University of Leeds, Leeds, United Kingdom, 31 Institute of Epidemiology I, Helmholtz Zentrum München-German Research Center for Environmental Health, Neuherberg, Germany, 
32 Institute of Epidemiology II, Helmholtz Zentrum München-German Research Center for Environmental Health, Neuherberg, Germany, 33 MRC-HPA Centre for Environment and Health, Department of Epidemiology and Biostatistics, School of Public Health, Imperial College London, London, United Kingdom, 34 Department of Obstetrics and Gynaecology, Institute of Clinical Sciences, University of Oulu, Oulu, Finland, 35 Department of Public Health, Hjelt Institute, University of Helsinki, Helsinki, Finland, $\mathbf{3 6}$ Institute of Biomedicine, University of Oulu, Oulu, Finland, 37 Department of Psychiatry, Kuopio University Hospital, Kuopio, Finland, $\mathbf{3 8}$ Centre for Paediatric Epidemiology and Biostatistics and Medical Research Council Centre for the Epidemiology of Child Health, University College London Institute of Child Health, London, United Kingdom, 39 Hannover Unified Biobank, Hannover Medical School, Hannover, Germany, 40 Research Unit of Molecular Epidemiology, Helmholtz Zentrum München-German Research Center for Environmental Health, Neuherberg, Germany, 41 Department of Social Services and Health Care, Jakobstad, Finland, 42 Folkhälsan Research Centre, Helsinki, Finland, 43 Department of Internal Medicine II-Cardiology, University of Ulm Medical Center, Ulm, Germany, 44 Finnish Institute of Occupational Health, Helsinki, Finland, 45 Steno Diabetes Center, Gentofte, Denmark, 46 Department of Mathematical Sciences, University of Oulu, Oulu, Finland, 47 Wellcome Trust Sanger Institute, Hinxton, United Kingdom, 48 Department of Children, Young People and Families, National Institute for Health and Welfare, Oulu, Finland, 49 Institute of Biometrics and Epidemiology, German Diabetes Center, Düsseldorf University, Düsseldorf, Germany, $\mathbf{5 0}$ Department of Internal Medicine, Erasmus Medical Center, Rotterdam, The Netherlands, $\mathbf{5 1}$ Institute of Diagnostics, University of Oulu, Oulu, Finland, $\mathbf{5 2}$ Department of Internal Medicine, Institute of Clinical Medicine, University of Oulu, Oulu, Finland, $\mathbf{5 3}$ Neuroscience Campus Amsterdam, Amsterdam, The Netherlands, $\mathbf{5 4}$ Department of Psychiatry, VU University Medical Center, Amsterdam, The Netherlands, $\mathbf{5 5}$ Molecular Medicine and Science for Life Laboratory, Department of Medical Sciences, Uppsala University, Uppsala, Sweden, 56 Department of Health Sciences, University of Leicester, Leicester, United Kingdom, $\mathbf{5 7}$ Centre for Public Health, Queen's University of Belfast, Belfast, Northern Ireland, $\mathbf{5 8}$ Department of Cardiology, Toulouse University School of Medicine, Rangueil Hospital, Toulouse, France, $\mathbf{5 9}$ Centre of Excellence for Public Health Northern Ireland, Queen's University of Belfast, Belfast, Northern Ireland, $\mathbf{6 0}$ Institute of Cardiometabolism and Nutrition, INSERM UMR S937, Pierre and Marie Curie University, Paris, France, $\mathbf{6 1}$ Department of Epidemiology and Public Health, University of Strasbourg, Strasbourg, France, $\mathbf{6 2}$ Institut Pasteur de Lille, INSERM U744, Université Lille Nord de France, Lille, France, 63 Epidemiology and Preventive Medicine Research Centre, Department of Clinical and Experimental Medicine, University of Insubria, Varese, Italy, 64 Department of Experimental Medicine, University of Milano-Bicocca, Monza, Italy, $\mathbf{6 5}$ Washington University School of Medicine, St Louis, Missouri, United States of America, $\mathbf{6 6}$ Population Studies Unit, Department of Chronic Disease Prevention, National Institute for Health and Welfare, Turku, Finland, $\mathbf{6 7}$ Department of Health, Functional Capacity and Welfare, National Institute for Health and Welfare, Helsinki, Finland, 68 Department of Clinical Genetics, Erasmus Medical Center, Rotterdam, The Netherlands, $\mathbf{6 9}$ Netherlands Genomic Initiative, Leiden, The Netherlands, $\mathbf{7 0}$ Centre for Medical Systems Biology, Leiden, The Netherlands, $\mathbf{7 1}$ MRC Centre for Integrative Epidemiology Unit, University of Bristol, Bristol, United Kingdom, 72 Department of Mental Health and Substance Abuse Services, National Institute for Health and Welfare, Helsinki, Finland, 73 Munich Heart Alliance, Munich, Germany, $\mathbf{7 4}$ Institute of Medical Informatics, Biometry and Epidemiology, Chair of Epidemiology, Ludwig-MaximiliansUniversität, Munich, Germany, 75 Klinikum Grosshadern, Munich, Germany, 76 Department of Medicine, Helsinki University Central Hospital, Helsinki, Finland, 77 Department of Medical Sciences, Uppsala University, Uppsala, Sweden, 78 Department of Epidemiology and Biostatistics, Imperial College London, London, United Kingdom, 79 Division of Welfare and Health Promotion, National Institute for Health and Welfare, Helsinki, Finland, 80 Faculty of Medicine, University of Iceland, Reykjavík, Iceland, $\mathbf{8 1}$ Oxford National Institute for Health Research Biomedical Research Centre, Churchill Hospital, Oxford, United Kingdom, 82 Department of Genomics of Common Disease, School of Public Health, Imperial College London, London, United Kingdom

\section{Abstract}

Background: The association between adiposity and cardiometabolic traits is well known from epidemiological studies. Whilst the causal relationship is clear for some of these traits, for others it is not. We aimed to determine whether adiposity is causally related to various cardiometabolic traits using the Mendelian randomization approach.

Methods and Findings: We used the adiposity-associated variant rs9939609 at the FTO locus as an instrumental variable (IV) for body mass index (BMI) in a Mendelian randomization design. Thirty-six population-based studies of individuals of European descent contributed to the analyses. Age- and sex-adjusted regression models were fitted to test for association between (i) rs9939609 and BMI $(n=198,502)$, (ii) rs9939609 and 24 traits, and (iii) BMI and 24 traits. The causal effect of BMI on the outcome measures was quantified by IV estimators. The estimators were compared to the BMI-trait associations derived from the same individuals. In the IV analysis, we demonstrated novel evidence for a causal relationship between adiposity and incident heart failure (hazard ratio, 1.19 per BMI-unit increase; $95 \% \mathrm{Cl}, 1.03-1.39$ ) and replicated earlier reports of a causal association with type 2 diabetes, metabolic syndrome, dyslipidemia, and hypertension (odds ratio for IV estimator, 1.1-1.4; all $p<0.05$ ). For quantitative traits, our results provide novel evidence for a causal effect of adiposity on the liver enzymes alanine aminotransferase and gamma-glutamyl transferase and confirm previous reports of a causal effect of adiposity on systolic and diastolic blood pressure, fasting insulin, 2-h post-load glucose from the oral glucose tolerance test, C-reactive protein, triglycerides, and high-density lipoprotein cholesterol levels (all $p<0.05$ ). The estimated causal effects were in agreement with traditional observational measures in all instances except for type 2 diabetes, where the causal estimate was larger than the observational estimate $(p=0.001)$.

Conclusions: We provide novel evidence for a causal relationship between adiposity and heart failure as well as between adiposity and increased liver enzymes.

Please see later in the article for the Editors' Summary. 
Citation: Fall T, Hägg S, Mägi R, Ploner A, Fischer K, et al. (2013) The Role of Adiposity in Cardiometabolic Traits: A Mendelian Randomization Analysis. PLoS Med 10(6): e1001474. doi:10.1371/journal.pmed.1001474

Academic Editor: Cosetta Minelli, Centre for Biomedicine, EURAC, Italy

Received December 3, 2012; Accepted May 14, 2013; Published June 25, 2013

Copyright: @ 2013 Fall et al. This is an open-access article distributed under the terms of the Creative Commons Attribution License, which permits unrestricted use, distribution, and reproduction in any medium, provided the original author and source are credited.

Funding: ENGAGE (European Network for Genetic and Genomic Epidemiology) Consortium, the European Community's Seventh Framework Programme grant FP7-HEALTH-F4-2007 (201413); Academy of Finland (104781, 120315, 129418, 139635, 141054), Center of Excellence in Complex Disease Genetics (213506, 129680), and SALVE research program (129418, 129494); Australian National Health and Medical Research Council (241944, 339462, 389927, 389875, 389891, 389892, 389938, 442915, 442981, 496739, 552485, 552498); Australian Research Council (A7960034, A79906588, A79801419, DP0770096, DP0212016, DP0343921); Avera Institute, Sioux Falls, South Dakota (USA); Biobanking and Biomolecular Resources Research Infrastructure (BBMRI -NL, 184.021.007); Biotechnology and Biological Sciences Research Council (BBSRC); British Heart Foundation; Center for Medical Systems Biology (CSMB, NWO Genomics); Center of Excellence in Genomics (EXCEGEN); Chronic Disease Research Foundation (CDRF); City of Malmö; Crafoord Foundation; Department of Health via the National Institute for Health Research (NIHR) comprehensive Biomedical Research Centre award to Guy's \& St Thomas' NHS Foundation Trust in partnership with King's College London; Development Fund of University of Tartu (SP1GVARENG); Diabetes Programme at the Lund University; Erasmus MC; Erasmus University Rotterdam; Estonian Government (SF0180142s08); Estonian Research Roadmap through Estonian Ministry of Education and Research (3.2.0304.11-0312); Estonian Science Foundation (EstSF ETF9353); EU 5th Framework Programme GenomEUtwin Project (QLG2-CT-2002-01254, EU/QLRT-2001-01254); EU Framework Programme 7 funding stream (IMI SUMMIT); EUR Fellowship; European Commission (EURO-BLCS, Framework 5 award QLG1-CT-2000-0164, FP6 STRP 018947, LSHG-CT-2006019473); European Community's Seventh Framework Program (FP7/2007-2013, CEED3 223211); European Community's Sixth Framework Programme Cardiogenics project (LSHM-CT-2006-037593); European Foundation for the Study of Diabetes (EFSD); European Science Council (ERC Advanced, 230374); European Science Foundation (ESF, EU/QLRT-2001-01254); European Union (EU/WLRT-2001-01254); EUROSPAN (European Special Populations Research Network); Faculty of Medicine, Lund University; Fellowship Schemes NBIC/BioAssist/RK (2008.024); Finnish Diabetes Research Society; Finnish Foundation for Cardiovascular Research; Folkhälsan Research Foundation; Foundation for Life and Health in Finland; Foundation for the US National Institutes of Health; French Institute of Health and Medical Research (U258); Genetic Association Information Network (GAIN); German Federal Ministry of Education and Research (BMBF); German National Genome Research Network (NGFNPlus, 01GS0834); German Research Center for Environmental Health; Guide Dogs for the blind Association(GDBA); Health Administration of Regione Lombardia (9783/1986, 41795/1993, 31737/1997, 17155/2004); Health Informatics Centre; Helmholtz Zentrum München; High Performance Computing Center of University of Tartu; International Agency for Research on Cancer; Internationale Stichting Alzheimer Onderzoek (ISAO); Jakobstad Hospital; K Medical Research Council; Knut and Alice Wallenberg Foundation; LMUinnovativ; Manpei Suzuki Diabetes Foundation; Medical Research Council, UK (G0500539, G0600705, PrevMetSyn/SALVE); Medical Society of Finland; Ministry of Education, Culture and Science, The Netherlands; Ministry of Health Welfare and Sports, The Netherlands; Munich Center of Health Sciences (MC Health); Municipality of Rotterdam; National Cancer Institute (N01-RC-37004); MyEuropia Research Training Network; National Institute for Health Research (NIHR) Leicester Cardiovascular Biomedical Research Unit; National Health and Medical Research Council (NHMRC); National Eye Institute via an NIH/CIDR genotyping project (R01EY018246-01-1 PI: Terri Young); National Institute of Aging (NIA); Netherlands Brain Foundation (HersenStichting Nederland); Netherlands Consortium for Healthy Aging (NCHA); (050-060-810); Netherlands Genomics Initiative (NGI); Netherlands Heart Foundation; Netherlands Organization for Health Research and Development (ZonMw) (10-000-1002, 904-61-090, 985-10-002, 904-61-193, 480-04-004, 400-05-717); Netherlands Organization for Scientific Research (NWO) (175.010.2005.011, 911-03-012, vici, 918-76-619, veni, 916.12.154 Addiction-31160008, Middelgroot-911-09-032, Spinozapremie 56-464-14192); Netherlands Scientific Organization (904-61-090, 904-61-193, 480-04-004, 400-05717, I 480-05-003); Neuroscience Campus Amsterdam (NCA); NHLBI (5R01HL087679-02); Novo Nordisk Foundation; Närpes Research Foundation; Ollqvist Foundation; Pfizer Global Research Awards for Nicotine Dependence (GRAND); Pfizer Pharmaceuticals; Påhlsson Foundation; Region Skåne; Research Institute for Diseases in the Elderly (014-93-015; RIDE; RIDE2); Royal Swedish Academy of Sciences; Rutgers University Cell and DNA Repository (NIMH U24 MH068457-06); Seventh Framework Programme ENGAGE project (HEALTH-F4-2007-201413); Signe and Ane Gyllenberg Foundation; Sigrid Juselius Foundation; STAMPEED program (1RL1MH083268-01); Swedish Cancer Society; Swedish Cultural Foundation in Finland; Swedish Diabetes Foundation; Swedish Diabetes Research Society; Swedish Foundation for Strategic Research (SSF; ICA08-0047); Swedish Heart and Lung Foundation; Swedish Medical Research Council; Swedish Ministry for Higher Education; Swedish Research Council for Medicine and Health (Linné grant 349-2008-6589, a strategic SFO grant, Exodiab 2009-1039, M-2005-1112, 2009-2298); Swedish Research Council for Infrastructures; Swedish Society of Medicine; US NIH (AA07535, AA10248, AA11998, AA13320, AA13321, AA13326, AA14041, AA15416, AA17688, DA12854, MH66206, R01D0042157-01A, AG028555, AG08724, AG04563, AG10175, AG08861, R01HL089650-02, DK U01-066134, 5R01MH63706:02, RO1 MH059160, 1RC2MH089951-01, 1RC2 MH089995-01); University Hospital Oulu; University of Dundee; University of Ulm; Uppsala University; Uppsala University Hospital; US National Heart, Lung and Blood Institute; US Public Health Service contracts (N01-CN-45165, N01-RC-45035); Vasa and Närpes Health centers; Wellcome Trust (Biomedical Collections Grant GR072960); Wellcome Trust Sanger Institute; VU University's Institute for Health and Care Research (EMGO+); TDS is an NIHR senior Investigator and is holder of an ERC Advanced Principal Investigator award; CJH is an NIHR Senior Research Fellow. The funders had no role in study design, data collection and analysis, decision to publish, or preparation of the manuscript.

Competing Interests: TF has received honoraria by MSD for lecturing. GT, SG, VSt, UT, and KS are employees of deCODE Genetics/Amgen, a biotechnology company. OHF is the recipient of a grant from Pfizer Nutrition to establish a new center of ageing research: ErasmusAGE KH received funding via the Finnish Academy (grant number 129418). JK holds grants from the EU FP7 (funding the present research and other projects), US NIH, the Academy of Finland, and several Finnish Foundations. JK consulted for Pfizer Inc. in 2012 on nicotine dependence. LG, GDS, and MIM are members of the Editorial Board of PLOS Medicine. All other authors have declared that no competing interests exist.

Abbreviations: ALT, alanine aminotransferase; BMI, body mass index; CRP, C-reactive protein; CHD, coronary heart disease; CVD, cardiovascular disease; GGT, gamma-glutamyl transferase; HbA1c, hemoglobin A1c; HDL-C, high-density-lipoprotein cholesterol; IL-6, interleukin-6; IV, instrumental variable; LD, linkage disequilibrium; LDL-C, low-density lipoprotein cholesterol; MR, Mendelian randomization; OGTT, oral glucose tolerance test; OR, odds ratio; SNP, single nucleotide polymorphism; T2D, type 2 diabetes.

*E-mail: erik.ingelsson@medsci.uu.se (EI); i.prokopenko@imperial.ac.uk (IP)

9 These authors contributed equally to this work.

- These authors are joint senior authors on this work. 


\section{Introduction}

The incidence and prevalence of cardiovascular disease (CVD) are continuously increasing in parallel with the increase in obesity and metabolic diseases, especially in low- and middle-income countries [1]. An association between increased body mass index (BMI) and cardiometabolic diseases has been demonstrated by many well-designed epidemiological studies, and has previously been shown to be close to log-linear, at least for BMI>25 kg/m [2]. However, confounding, reverse causation, and other issues with conventional observational studies can seriously impair the possibility of making causal inference, and lead to imprecision in estimation of both the direction and magnitude of the effects, as has been shown for the associations between BMI and mortality from respiratory disease and lung cancer [3]. Several randomized clinical trials have found that lifestyle interventions aiming at weight loss decrease the risk of type 2 diabetes (T2D) and metabolic syndrome [4-6], whereas the follow-ups of these studies for GVD outcomes have been underpowered [7,8]. The causal relationships of long-term obesity to disease are difficult to assess within conventional randomized clinical trials, necessitating other study designs.

In the past decade, instrumental variable (IV) analysis has become widely used for assessing causality using genetic variants under the name of "Mendelian randomization" (MR) [9]. MR represents one of the methods to infer causal relationships between epidemiologically relevant phenotypes. In MR study designs, a genetic variant associated with an intermediate phenotype (in the present report, BMI) is used as an IV to evaluate the causal relationship of the intermediate phenotype with the outcome of interest (Figure 1). Since genetic variants are assumed to be randomly distributed within a population, the IV is regarded as independent of confounders affecting the intermediate phenotype (BMI)-outcome relationship [10]. In the presence of confounding and reverse causation, the IV approach is an alternative for statistical estimation of causal relationships, especially within large-scale studies, where classical epidemiological modeling - fully adjusted for a wide range of covariates and across numerous outcomes - would be difficult. While acknowledging the issue of observed and unobserved confounding, we consider MR as a pragmatic tool for elucidating the epidemiological data through utilization of the findings from genetic association studies on intermediate phenotypes. The strength of the causal interpretation depends crucially on the validity of assumptions and caveats within MR experiments, some of which are difficult to evaluate [11]. If the basic assumptions are violated, invalid conclusions would be drawn from the experiments. In the past five years, large-scale collaborative efforts have successfully identified more than 30 loci associated with BMI and obesity [12]. The single nucleotide polymorphism (SNP) rs9939609, within the fat-mass- and obesity-associated gene (FTO) locus, was the first associated with BMI by genome-wide association studies, and the association has been extensively replicated in individuals of European descent and in other ethnic groups [12]. FTO locus variants alone have been reported to explain $0.34 \%$ of the phenotypic variability in BMI [13], and the rs9939609 variant is considered a good instrument in MR studies because of its specificity (lack of known pleiotropy) and decent effect size $[14,15]$.

Several MR studies using FTO variants have supported the hypothesis of a causal relationship between adiposity and cardiometabolic phenotypes, such as ischemic heart disease, Creactive protein (CRP), systolic and diastolic blood pressure, fasting insulin, triglycerides, metabolic syndrome, and decreased concentrations of high-density lipoprotein cholesterol (HDL-C) [14-19]. However, the causal relationship between obesity and increased risk of other CVD and metabolic phenotypes, such as heart failure, stroke, and non-alcoholic fatty liver disease, is not yet established using these methods, probably because of power issues, as large sample sizes are needed for MR studies [15]. Table 1 shows an overview of previous MR studies of adiposity and cardiometabolic phenotypes, with reported sample sizes and instruments used.

In the present investigation, which is the largest MR study to date, we aimed to evaluate the evidence for a causal relationship between adiposity, assessed as elevated BMI, and a wide range of cardiometabolic phenotypes including coronary heart disease, stroke, T2D, and heart failure, as well as a number of intermediate phenotypes related to future disease end points.

\section{Methods}

The study was conducted within the European Network for Genetic and Genomic Epidemiology (ENGAGE) consortium, represented here by 36 cross-sectional and longitudinal cohort studies and up to 198,502 individuals of European descent (Table S1).

\section{Genotypes}

Of the many highly correlated variants within the FTO locus, we chose the widely confirmed and extensively studied variant rs9939609 as the index SNP and IV for this study. Whenever possible, we used direct genotype information for rs9939609 from participating cohorts $(n=21)$ that had FTO variant genotypes available (Table S2). Eleven out of 36 studies performed de novo genotyping of rs9939609 for the present study, and ten studies used direct genotype information on rs9939609 from previously genotyped array data. Whenever rs9939609 was not genotyped directly, we used either (i) the HapMap II CEU (European) reference panel-imputed genetic information from genome-wide association studies (http://hapmap.ncbi.nlm.nih.gov/downloads/ genotypes/2008-10_phaseII/) for rs9939609 $(n=5)$ or (ii) genotype information from a predefined list of proxies that are in high linkage disequilibrium (LD) with rs9939609 $\left(n=10, r^{2}>0.9\right.$; Table S3). For the remaining studies, we used the directly genotyped proxies rs1 $1075989\left(n=5, r^{2}=1.0\right), \operatorname{rs} 3751812\left(n=4, r^{2}=1.0\right)$, and rs $1421085\left(n=1, r^{2}=0.93\right)$. We estimated effects of the BMIincreasing A allele of rs9939609, or for the corresponding alleles from proxies (using HapMap II CEU LD data), on phenotypes. We excluded individuals from analysis when the overall array sample call rate was $<95 \%$. All studies reported SNPs with HardyWeinberg equilibrium exact $p>0.0001$, an information content $>0.99$ for imputed SNPs, and a call rate>0.95 for genotyped SNPs.

\section{Outcomes}

We studied nine dichotomous cardiometabolic outcomes in up to 160,347 individuals and 14 quantitative cardiometabolic traits in up to 147,644 individuals. Only individuals with both BMI and FTO genotype information available were included in the study.

The CVD dichotomous outcomes of interest were coronary heart disease (CHD), heart failure, hemorrhagic stroke, ischemic stroke, all-cause stroke, and hypertension diagnosed at any time point (ever) during the life course (Table 2). The metabolic dichotomous outcomes included dyslipidemia, metabolic syndrome, and T2D diagnosed at any time point (ever) during the life course. The diagnoses of $\mathrm{CHD}$, heart failure, hemorrhagic stroke, ischemic stroke, all-cause stroke, and all-cause mortality 


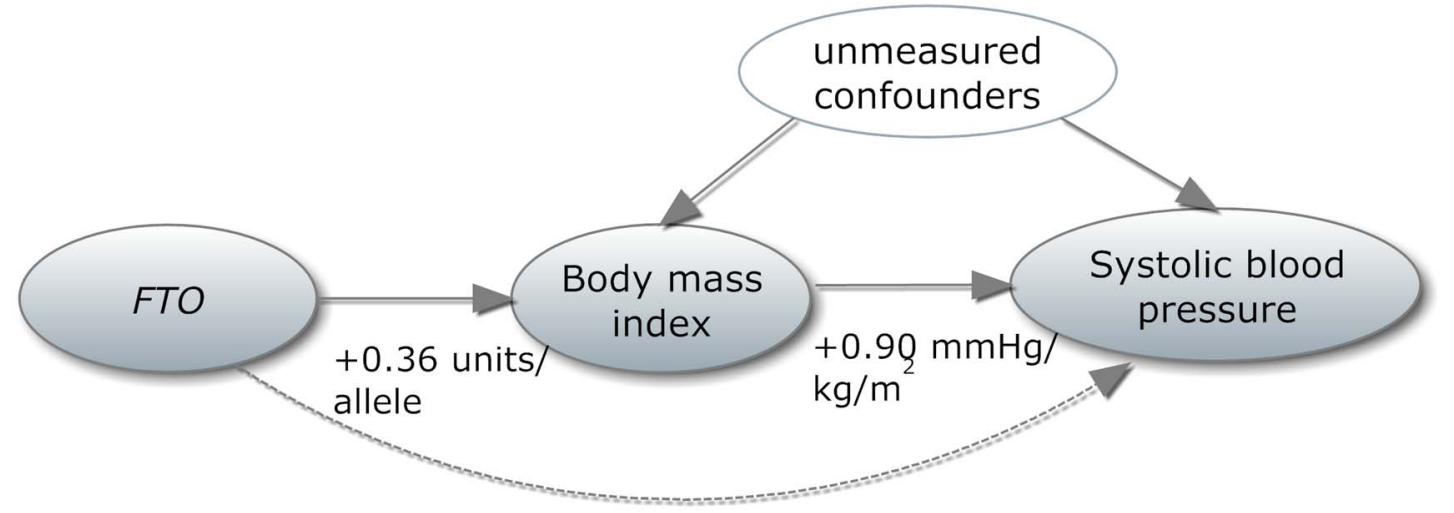

+0.32 mmHg/BMI-increasing allele

IV estimator for causal relationship of BMI and systolic blood pressure: $+0.89 \mathrm{mmHg} /\left(\mathrm{kg} / \mathrm{m}^{2}\right)$

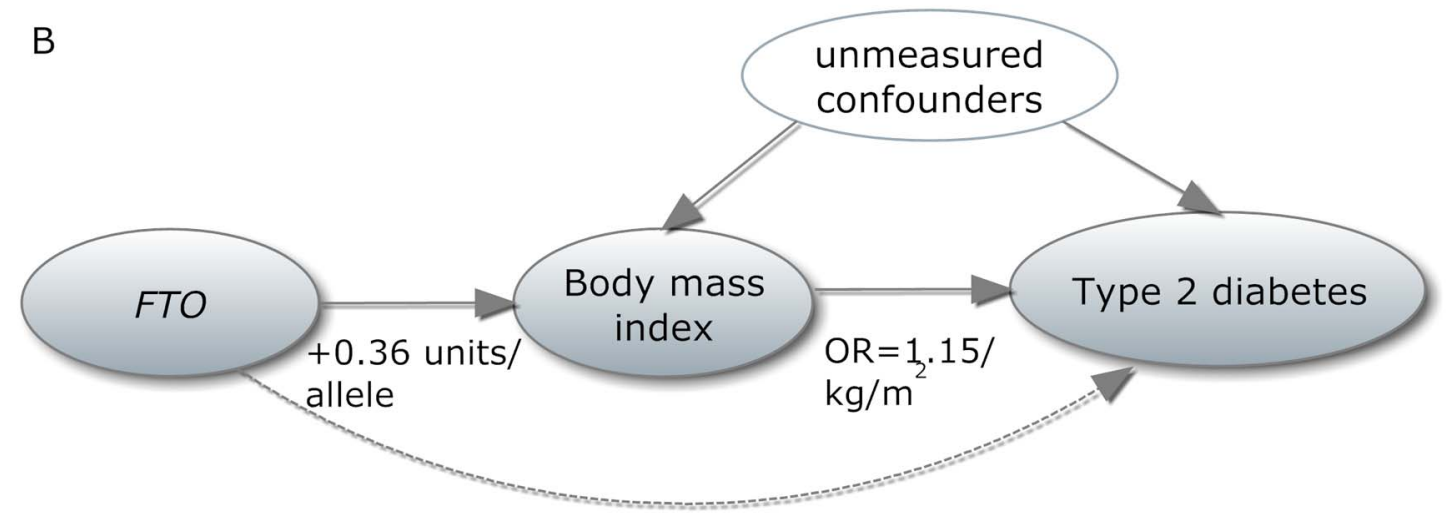

$\mathrm{OR}=1.12 / \mathrm{BMI}$-increasing allele
IV estimator for causal relationship of BMI and Type 2 diąbetes $\mathrm{OR}=1.37 /\left(\mathrm{kg} / \mathrm{m}^{2}\right)$

Figure 1. In a Mendelian randomization framework, genotype-phenotype association is assumed to be independent of confounding factors. (A) In an example from our study, the IV estimator is calculated as the beta coefficient from the association of FTO with systolic blood pressure divided by the beta coefficient from the association of $F T O$ with BMI (IV estimator $=0.32 / 0.36=0.89 \mathrm{~mm} \mathrm{Hg} / \mathrm{BMI}$ unit). The IV estimator is equivalent to what is seen when systolic blood pressure is regressed on BMI. These results are supportive of a causal, non-confounded relationship. For binary traits, the calculation of the IV estimator is done on the log-odds scale. (B) The relationship of BMI with T2D, where the IV estimator is $\ln \left(\mathrm{OR}_{\mathrm{IV}}\right)=\ln (1.12) / 0.36$, which equals a causal OR of $\mathrm{BMI}$ for T2D of 1.37. This is larger than what is seen in the standard age- and sexadjusted logistic regression of T2D on BMI $(p=0.001)$, indicating that confounding or reverse causation may be present or that BMI measured once in adulthood does not fully reflect the effect of lifetime adiposity. doi:10.1371/journal.pmed.1001474.g001

were based on health registries and/or validated medical records (Table S4). Hypertension, dyslipidemia, and T2D diagnoses could be self-reported or based on biochemical measurement within the study, in addition to health registries and validated medical records (Table S4). The diagnosis of metabolic syndrome was based on a modified National Cholesterol Education Program Adult Treatment Panel III definition [20]. We analyzed a subset of individuals with prospectively collected events available for incident cases of all binary outcomes and for all-cause mortality as outcome.
We studied the following quantitative phenotypes (Table 3): (i) measurements of glucose homeostasis in individuals without diabetes: fasting glucose, 2-h post-load glucose from the oral glucose tolerance test (OGTT), hemoglobin Alc (HbAlc), and fasting insulin; (ii) diastolic and systolic blood pressure, with adjustment for blood pressure medication; (iii) lipid metabolism (in individuals without lipid-lowering medication): HDL-C, lowdensity lipoprotein cholesterol (LDL-C), total cholesterol, and triglycerides; (iv) liver enzyme activity and leakage: alanine aminotransferase (ALT) and gamma-glutamyl transferase (GGT); 
Table 1. Comparison of our study with previous Mendelian randomization studies of adiposity on cardiometabolic phenotypes.

\begin{tabular}{|c|c|c|c|c|c|c|c|c|}
\hline \multirow[t]{2}{*}{ Phenotype } & \multicolumn{3}{|c|}{ Present Study Using FTO as Instrument } & \multicolumn{5}{|c|}{ Previous Studies } \\
\hline & $N$ Total & $N$ Cases & $\begin{array}{l}\text { Evidence for } \\
\text { Causality? }\end{array}$ & $N$ Total & $N$ Cases & $\begin{array}{l}\text { Evidence for } \\
\text { Causality? }\end{array}$ & Reference & $\begin{array}{l}\text { Instrument Other } \\
\text { than FTO Only }\end{array}$ \\
\hline CHD & 119,630 & 10,372 & - & 75,627 & 11,056 & + & [16] & FTO, MC4R, TMEM18 \\
\hline Heart failure & 75,770 & 6,068 & + & & & & N.A. & \\
\hline Hemorrhagic stroke & 77,020 & 588 & - & & & & N.A. & \\
\hline Ischemic stroke & 106,402 & 4,233 & - & & & & N.A. & \\
\hline Stroke & 85,175 & 4,003 & - & & & & N.A. & \\
\hline T2D & 160,347 & 20,804 & + & & & & $-^{\mathrm{a}}$ & \\
\hline Dyslipidemia & 96,380 & 33,414 & + & & & & N.A. & \\
\hline Hypertension & 155,191 & 56,721 & + & 37,027 & 24,813 & + & [18] & FTO, MC4R \\
\hline Metabolic syndrome & 49,592 & 11,608 & + & 12,555 & N.A. & + & [15] & \\
\hline Mortality & 68,762 & 8,640 & - & & & & N.A. & \\
\hline 2-h post-OGTT glucose & 21,257 & & + & & & & N.A. & \\
\hline \multirow[t]{2}{*}{ Fasting glucose } & 84,910 & & - & 13,632 & & + & [15] & \\
\hline & & & & 2,230 & & + & [17] & \\
\hline $\mathrm{HbA} 1 \mathrm{c}$ & 35,471 & & - & 8,876 & & - & [15] & \\
\hline \multirow[t]{2}{*}{ Fasting insulin } & 48,018 & & + & 12,095 & & + & [15] & \\
\hline & & & & 2,229 & & - & [17] & \\
\hline \multirow[t]{2}{*}{ Diastolic blood pressure } & 130,380 & & + & 15,619 & & - & [15] & \\
\hline & & & & 37,010 & & + & [18] & FTO, MC4R \\
\hline \multirow[t]{3}{*}{ Systolic blood pressure } & 147,644 & & + & 15,624 & & - & [15] & \\
\hline & & & & 37,011 & & + & [18] & FTO, MC4R \\
\hline & & & & 2,204 & & + & [17] & \\
\hline \multirow[t]{2}{*}{$\mathrm{HDL}-\mathrm{C}$} & 132,782 & & + & 13,659 & & + & [15] & \\
\hline & & & & 2,224 & & - & [17] & \\
\hline \multirow[t]{2}{*}{ LDL-C } & 123,026 & & - & 13,476 & & - & [15] & \\
\hline & & & & 2,224 & & - & [17] & \\
\hline ALT & 46,754 & & + & 6,171 & & - & [15] & \\
\hline \multirow[t]{3}{*}{ CRP } & 91,337 & & + & 21,836 & & + & [18] & \\
\hline & & & & 2,133 & & - & [17] & \\
\hline & & & & 5,804 & & + & [19] & FTO, MC4R \\
\hline GGT & 71,118 & & + & 6,596 & & - & [15] & \\
\hline IL-6 & 11,225 & & - & & & & N.A. & \\
\hline \multirow[t]{2}{*}{ Triglycerides } & 139,241 & & + & 13,651 & & + & [15] & \\
\hline & & & & 2,228 & & - & [17] & \\
\hline Total cholesterol & 147,619 & & - & 2,226 & & - & [17] & \\
\hline
\end{tabular}

a No formal MR study, although the association of FTO and T2D is well known. N.A, not applicable.

doi:10.1371/journal.pmed.1001474.t001

and (v) inflammation markers: CRP and interleukin-6 (IL-6). Prior to analysis the following variables were transformed to the natural logarithmic scale: fasting insulin, ALT, GGT, CRP, IL-6, and triglycerides (assay specifications are reported in Table S5).

\section{Statistical Analyses}

Association analyses. We assessed associations between the dichotomous outcomes and (i) FTO and (ii) BMI in each cohort using sex- and age-adjusted logistic regression models. We used Cox proportional hazards models to assess FTO and BMI associations with prospectively collected events [21]. The time origin in the present analysis was set to the date of first BMI measurement available. We assumed log-additive genetic effects on binary traits. We evaluated the associations of (i) FTO and (ii) $\mathrm{BMI}$ with the quantitative traits, as well as the association between FTO and BMI, using sex- and age-adjusted linear regression in each cohort, assuming an additive effect of the number of A alleles. The models are described in detail in Text S1. The software used for statistical analysis within each cohort is listed in Table S1.

Meta-analyses. As initial attempts at fixed-effects inversevariance-weighted meta-analysis indicated considerable betweencohort heterogeneity, we performed random-effects meta-analyses, leading to essentially unchanged effect estimates, but somewhat more conservative confidence intervals (Figure S1). Hence, all 


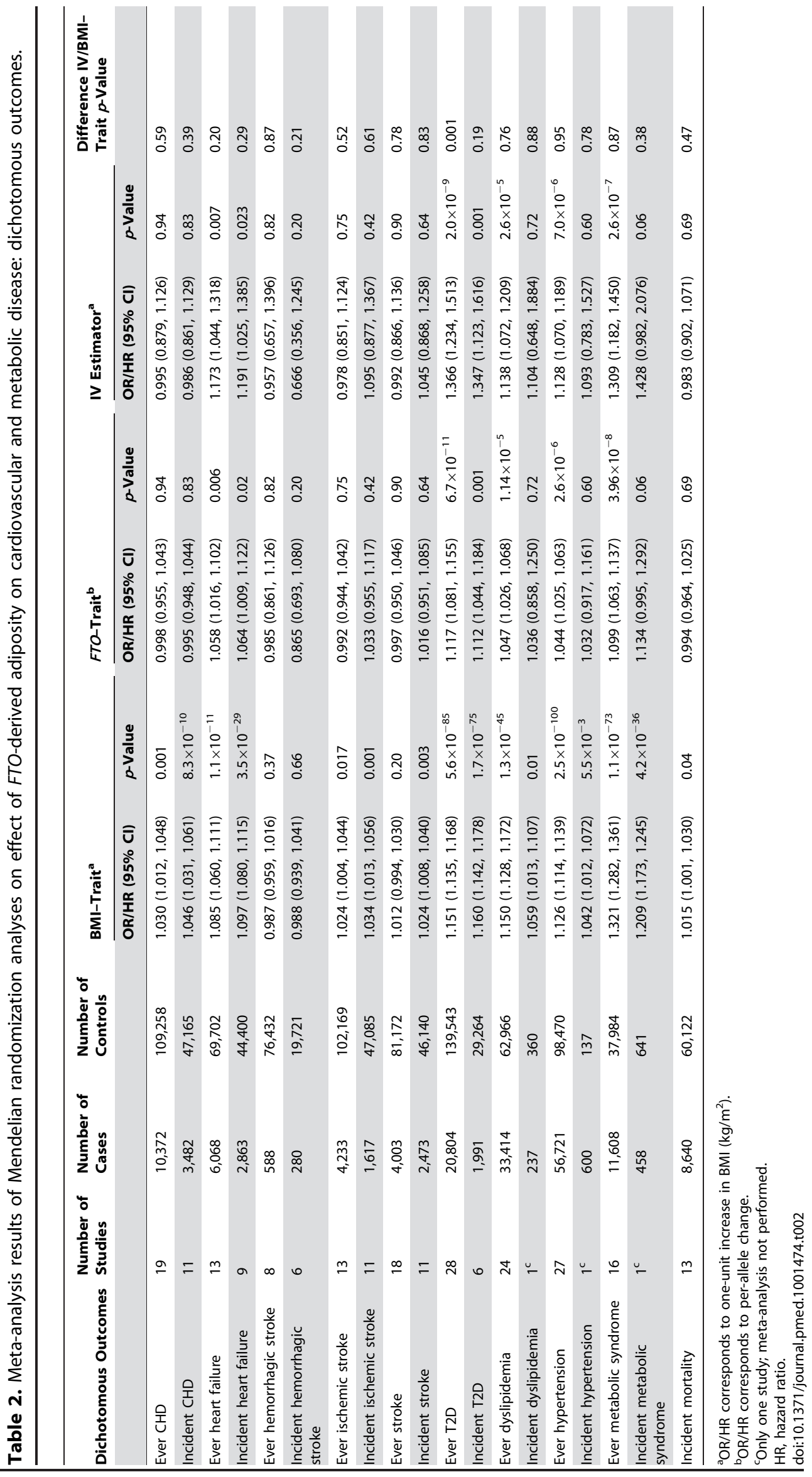




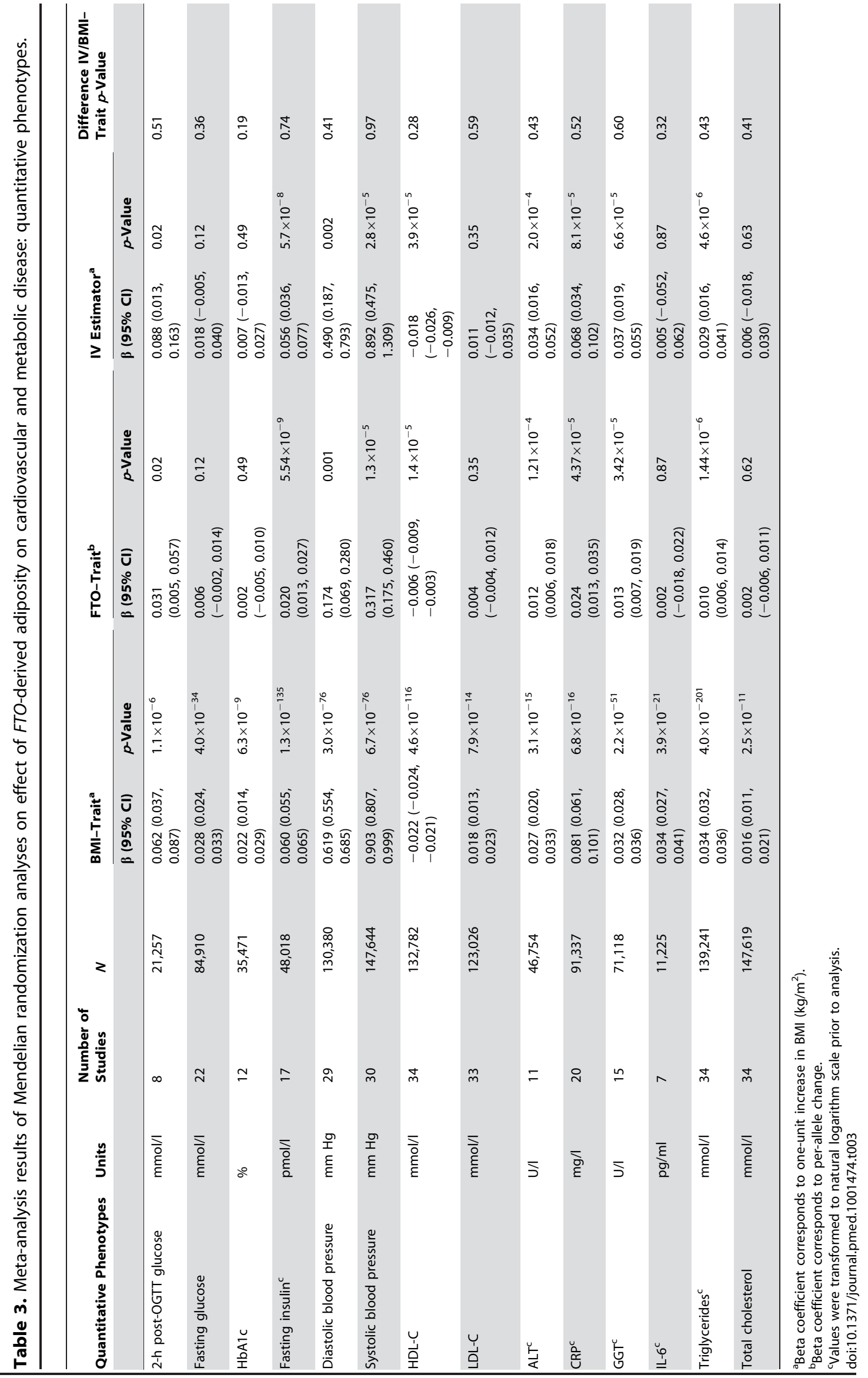


results presented are from random-effects meta-analysis. Analyses were run at two centers in parallel using different software packages (GWAMA and R) [22,23] and yielding identical results.

Instrumental variable analyses. We used the IV estimators to quantify the strength of the causal association between BMI and cardiometabolic traits. The estimate was found as a ratio between the two regression coefficients determined from association metaanalyses (Equation 1): estimated FTO effect on the given trait and estimated FTO effect on BMI in the full study sample $(n=198,502)$. For binary traits, the formula is identical to the Wald estimator [24].

$$
\beta_{\text {IV estimator }}=\frac{\beta_{\mathrm{FTO}-\mathrm{TRAIT}}}{\beta_{\mathrm{FTO}-\mathrm{BMI}}}
$$

For quantitative and binary outcomes with only one SNP as instrument, the IV estimator derived by Equation 1 is identical to that derived by the widely used two-stage least squares method [25]. The standard errors for the IV estimators were estimated using the delta method (Equation 2), ignoring correlation, based on a comprehensive sensitivity analysis; see Text S1, Figure S2, and Tables S6 and Table S7 for further details.

$$
\operatorname{se}_{\mathrm{IV}}=\operatorname{abs}\left(\beta_{\mathrm{IV}}\right) \sqrt{\left(\frac{\text { se }_{\mathrm{FTO}-\mathrm{BMI}}}{\beta_{\text {FTO-BMI }}}\right)^{2}+\left(\frac{\text { se }_{\mathrm{FTO}-\mathrm{TRAIT}}}{\beta_{\mathrm{FTO}}}\right)^{2}}
$$

For each trait, we tested the null hypothesis of no difference between the respective IV estimator and the conventional regression-based estimator of the effect of BMI on trait via a classical $z$-test.

We did not apply correction for multiple testing as the associations between BMI and multiple cardiometabolic traits are widely reported $[2,5]$.

\section{Results}

\section{Association between FTO Variant and BMI}

Random-effects meta-analysis of the association between FTO variant and BMI in the 36 studies $(n=198,502)$ showed a positive effect of the A allele on BMI ( $\beta=0.36$ per additional A allele; $95 \%$ CI, $\left.0.31-0.40 ; p=4.3 \times 10^{-52}\right)$, with an effect size in line with that of previous studies [13]. The effect estimates ranged between 0.05 and 0.74 BMI units per copy of A allele, yielding an $I^{2}$ for heterogeneity between studies of $55 \%\left(p=3.6 \times 10^{-5}\right.$; Figure 2; Text S1). We assessed potential causes of this heterogeneity in a meta-regression of the study-specific beta coefficient estimates of effect sizes for the association between FTO and BMI-including study-specific mean age and mean BMI as covariates-and whether the study was exclusively of a diabetes case group or not. Effect size estimates decreased non-significantly with increasing cohort age in cohorts with mean age $>40 \mathrm{y}(n=31, p=0.07)$.

\section{Associations between BMI and Cardiometabolic Traits}

We observed positive associations (all $p<0.05$ ) between BMI and ever and incident heart failure (Figure 3), ever and incident CHD, ever all-cause stroke, ischemic stroke, hypertension, dyslipidemia, metabolic syndrome, T2D, and mortality (Table 2). We did not observe an association between BMI and ever or incident hemorrhagic stroke, or incident all-cause stroke. BMI was associated (all $p<10^{-6}$ ) with all quantitative phenotypes: (i) fasting glucose, fasting insulin, 2-h post-OGTT glucose, and HbAlc; (ii) diastolic and systolic blood pressure; (iii) HDL-C, LDL-C, total cholesterol, and triglycerides; (iv) ALT and GGT; and (v) GRP and IL-6 (Table 3).

\section{Associations between FTO Variant and Cardiometabolic Traits}

We detected a novel association between the BMI-increasing allele of the FTO variant and increased odds/hazard ratios of ever and incident heart failure (Figure 4; Table 2). Associations (all $p<0.001)$ were observed between the FTO variant and increased odds/hazard ratios of ever or incident T2D, ever dyslipidemia, ever metabolic syndrome, and ever hypertension. The FTO variant was associated (all $p<0.05$ ) with increased levels of 2-h post-OGTT glucose, fasting insulin, diastolic blood pressure, systolic blood pressure, triglycerides, ALT, GGT, GRP, and decreased HDL-C.

\section{Instrumental Variable Analysis}

We identified at least nominally significant $(p<0.05)$ causal estimates for the effect of BMI (IV estimators) on ever and incident heart failure, ever hypertension, ever and incident T2D, ever dyslipidemia, and ever metabolic syndrome (Table 2). For other dichotomous outcomes, we were not able to confirm the presence of a causal effect of BMI using the IV approach. The estimates derived from IV analysis based on either logistic regression modeling or Cox proportional hazards models were similar for our significant findings.

The IV estimators pointed to a causal effect of higher BMI on an increase in (i) ALT and GGT levels, a novel finding from the present study; (ii) 2-h post-OGTT glucose and fasting insulin; and (iii) diastolic blood pressure and systolic blood pressure. We also observed an unfavorable effect of BMI on lipid metabolism (in individuals without lipid medication), as indicated by decreased levels of HDL-C and increased levels of triglycerides. The IV estimators pointed to a causal link between BMI and inflammation, as indicated by increased levels of CRP. We did not observe a causal effect of BMI on levels of fasting glucose, HbAlc, LDL-C, IL-6, or total cholesterol (Table 3).

Post hoc power calculation showed that for the binary traits with non-significant IVs (CHD, ischemic stroke, and all-cause stroke), we had an $80 \%$ chance of detecting an IV estimator odds ratio (OR) of 1.08-1.09/BMI unit or higher, and a 95\% chance of detecting an OR of 1.13-1.15/BMI unit or higher. For fasting glucose, we had a $80 \%$ chance of detecting a $0.014 \mathrm{mmol} / \mathrm{l}$ change per BMI unit and a $95 \%$ chance of detecting a $0.022 \mathrm{mmol} / \mathrm{l}$ change, smaller than the effect estimate from ordinary linear regression of BMI on glucose (0.028; Table 3).

The causal estimate of the relationship between BMI and ever T2D derived from the MR analysis (the IV estimator) (OR 1.37; $95 \%$ CI, 1.23-1.51) was different from the observed association between BMI and ever T2D (OR 1.15; 95\% CI, 1.14-1.17; $p=0.001)$.

\section{Discussion}

\section{Main Findings}

In this large-scale meta-analysis, we used a MR design to examine causal associations between adiposity, assessed as elevated BMI, and a number of cardiometabolic outcomes. The present study is, to our knowledge, the most comprehensive MR study published to date, including 24 traits in up to 198,502 individuals with FTO genotype and BMI information available. This analysis has enabled us to provide evidence for many biologically plausible causal relationships, such as those between adiposity and 


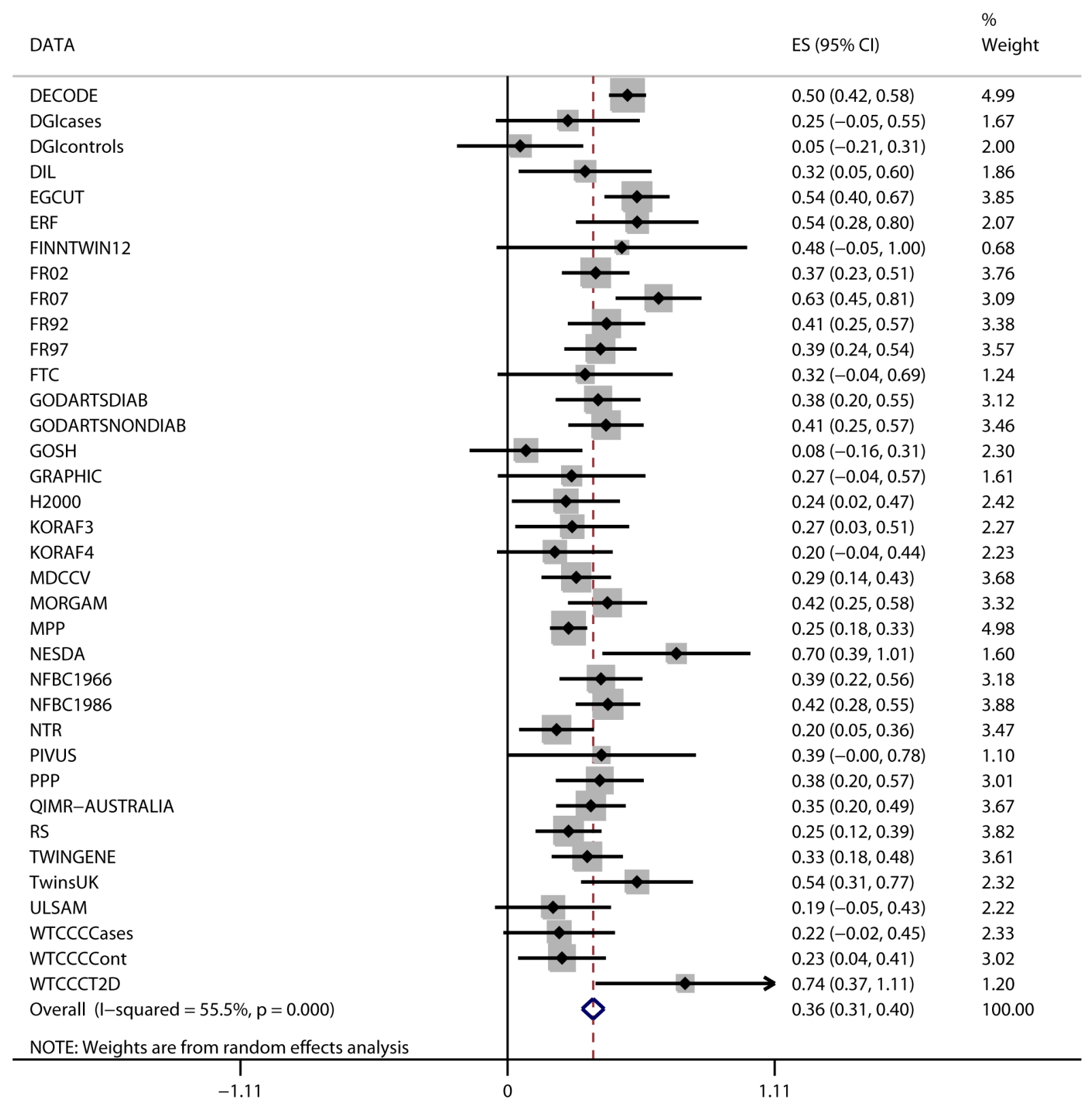

Figure 2. Association between FTO variant rs9939609 and BMI in 198,502 individuals. The assigned weight for each study in the metaanalysis is shown in percent (\% Weight). ES, estimate. For cohort abbreviations and references, see Table S1.

doi:10.1371/journal.pmed.1001474.g002

hypertension, and between adiposity and dyslipidemia. Furthermore, we demonstrated evidence for a causal relationship between (i) adiposity and heart failure and (ii) adiposity and increased concentrations of the liver enzymes ALT and GGT. In addition, we showed that traditional cross-sectional estimates of the BMI effect on T2D are smaller than the causal estimates of the BMIT2D relationship based on FTO-predicted obesity (IV analyses). This difference is probably driven by lifetime changes in BMI affecting T2D risk, and their attenuation introduced by a single measurement of BMI.

\section{Comparison with Previous MR Studies}

In the present population-based investigation, we confirm earlier findings that FTO-mediated adiposity increases the risk of metabolic syndrome and of increased CRP, fasting insulin, and triglyceride levels; increased systolic and diastolic blood pressure; and decreased concentrations of HDL-C [14,15,17-19].

Using standard regression methods for the association between BMI and other cardiovascular traits, we confirmed associations between adiposity and CHD, ischemic stroke, and all-cause stroke, but did not find an association with hemorrhagic stroke, where we had relatively few cases available for analyses. We could not demonstrate a causal relationship via IV methods applied to these cardiovascular outcomes. The same was true for several metabolic traits, such as for fasting glucose, HbAlc, IL-6, total cholesterol, and LDL-C. However, our findings do not exclude causal relationships as such, since despite the large study sample, the IV analyses brought estimators with rather wide confidence 


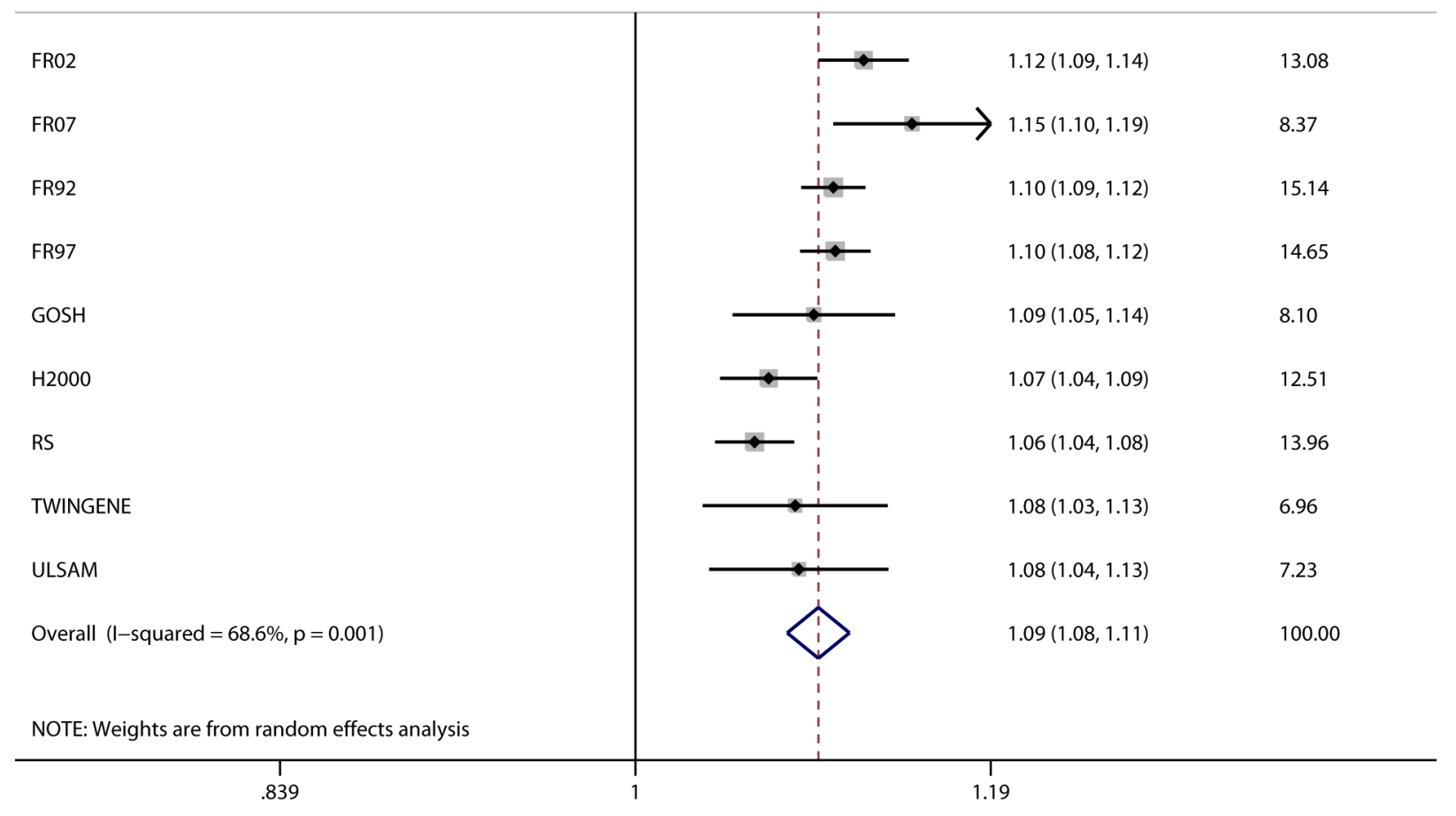

Figure 3. Association between BMI and incident heart failure in $\mathbf{2 , 8 6 3}$ cases and $\mathbf{4 4 , 4 0 0}$ controls. Estimates (ES) are shown on a hazard ratio scale for a one-unit increase in BMI. The assigned weight for each study in the meta-analysis is shown in percent (\% Weight). For cohort abbreviations and references, see Table S1.

doi:10.1371/journal.pmed.1001474.g003

intervals, a common feature when only one genotype is used as an IV. Our calculations showed low power to detect ORs of less than 1.05 in the present study, observed for several BMI-trait associations among those with non-significant IV estimators. We could not find evidence for a causal association between adiposity and all-cause mortality. While the causal association between these phenotypes might be absent, nonlinear relationships, potential survival bias, or low power due to a heterogeneous phenotype could have also affected the results.

We were not able to replicate the findings by Nordestgaard et al., who studied the association between adiposity and CHD using a combined allele score based on FTO, MC4R, and TMEM18 variants as an instrument for adiposity, and demonstrated a causal link between BMI and CHD risk [16]. Although the sample sizes and diagnostic criteria were comparable between that study and the present one, Nordestgaard et al. presented more precise estimates, which was probably primarily an effect of the stronger instrument, but the increased precision may also have been influenced by the notion that the ascertainment of CHD events was validated in the three cohorts included, and that results showed low heterogeneity. We found that the IV estimate for the effect of BMI on T2D was higher than that derived from standard logistic regression, which is similar to the finding of $\mathrm{Li}$ et al., conducted in east and south Asians [26]. Possible explanations of such an observation include the following: the cross-sectional nature of data that could result in reverse causation (weight loss due to disease or lifestyle interventions), and the notion that the lifelong effect of FTO on adiposity is not entirely captured by a single BMI measurement [27].

\section{Adiposity and Heart Failure}

We have provided evidence that the previously suggested association of adiposity with heart failure [28] may indeed be causal. A causal relationship may be mediated through effects of obesity on hypertension, dyslipidemia, and insulin resistance, associations that are also supported by our study. Hypertension, insulin resistance, and T2D have been independently associated with increased risk of heart failure [29,30]. Hypertension, T2D, dyslipidemia, and insulin resistance are also important risk factors for myocardial infarction, which often results in heart failure [31]. Additionally, increased BMI is associated with cardiac remodeling [32], possibly owing to increased hemodynamic load and increased oxidative stress [33]. Animal models have independently suggested direct apoptotic effects of adiposity on the myocardium [34]. Our study estimates the causal impact of a one-unit increase in $\mathrm{BMI}$ as a $17 \%$ increase in heart failure incidence. Extrapolating this estimate to the population level based on incidence rates reported by the World Health Organization [35] and the American Heart Association [36], a one-unit increase in BMI corresponds to roughly 220,000 additional heart failure cases in Europe and 113,000 additional cases in the US, at extensive costs for society.

\section{Adiposity and Liver Enzymes}

The higher concentrations of liver enzymes observed in the present study caused by an increased BMI are likely to be related to non-alcoholic fatty liver disease, which is characterized by lipid accumulation within hepatocytes as a consequence of increased levels of fatty acids in insulin-resistant individuals. This accumu- 


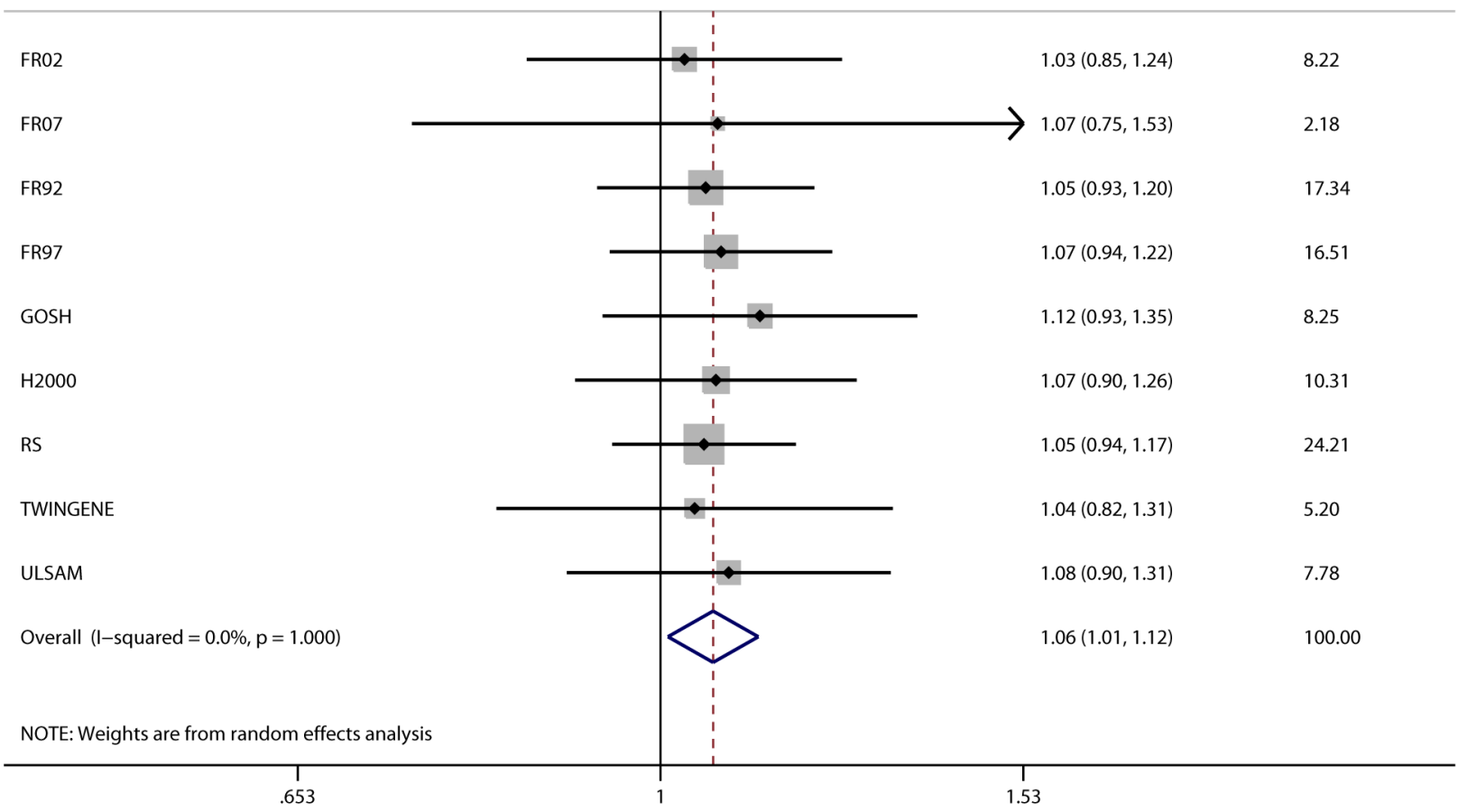

Figure 4. Association between $\mathbf{F T O}$ and incident heart failure in $\mathbf{2 , 8 6 3}$ cases and $\mathbf{4 4 , 4 0 0}$ controls. Estimates (ES) are shown on a hazard ratio scale per number of effect alleles. The assigned weight for each study in the meta-analysis is shown in percent (\% Weight). For cohort abbreviations and references, see Table S1.

doi:10.1371/journal.pmed.1001474.g004

lation predisposes to overproduction of reactive oxygen species, endoplasmic reticulum stress, and lipotoxicity, all of which are harmful to the hepatocytes [37].

\section{Strengths and Limitations}

The main strengths of the present investigation include the combination of the very large study sample, prospectively collected events, and a wide range of cardiometabolic phenotypes. The limitations of our study are tied to the validity of the assumptions underlying causal interpretation within MR studies. There are three main assumptions for a MR study: (i) independence between the instrument and confounders, i.e., FTO genotypes are randomized, (ii) a reliable association between the genetic variant and intermediate phenotype, and (iii) conditional independence between the genetic variant and the outcome, given the intermediate phenotype and the confounders, i.e., no pleiotropy [38]. Possible violations of the first and the third assumptions include population stratification, pleiotropic effects, canalization, epigenetic effects, and the presence of genes associated with confounders and outcomes in LD with the FTO variant. Neither the first nor the third assumption can be tested statistically in the observed data using single genotypes as the IV, and conclusions about such assumptions have to be based on previous biological knowledge. There are additional assumptions of MR studies regarding the quantification of the causal effect (as opposed to testing only; see Text $\mathrm{S} 1$ ).

The random distribution of genotypes in the population is the very basis of $M R$ and could be violated if separate ethnic groups with different allele frequencies were analyzed together without accounting for the population substructure. In the present study, all association analysis was done within each study (including individuals from a similar genetic background) separately, and all studies included only individuals of European ancestry. Hence, bias from population stratification is deemed unlikely [39].

With regards to the possibility of pleiotropic effects by FTO or genes in high LD with FTO, we acknowledge that although FTO is one of the most well-studied obesity loci, and there are credible hypotheses for its action on adiposity by increasing the appetite $[40,41]$, the precise mechanism of the FTO polymorphisms is still unclear, and potential pleiotropy cannot completely be ruled out. It has, however, been demonstrated previously that FTO is not associated with the most obvious potential confounders, such as smoking and drinking habits, income, or education [16]. A suggested way to assess pleiotropy in IV studies using multiple genotypes is to compare IV estimates between variants: if they are similar, it is less plausible that LD or pleiotropy is present [42]. This was done in the study by Nordestgaard and colleagues on the adiposity effect on CHD, and no difference between FTO, MC4R, and TMEM18 was seen in effect on CHD risk [16].

Concerning the reliability of the association (second assumption) between rs9939609 and BMI, this association has been widely replicated in many studies and populations [13,43,44]. While having the largest effect on BMI among known common variants, FTO constitutes a relatively weak instrument and thus results in wide confidence intervals for the IV estimators, despite the very large sample size. An approach to increase power in future studies would be to use multiple genetic variants as an instrument. In the present study, there is a possibility of introduction of a bias by 
using weak instruments in the calculation of the Wald estimator of dichotomous traits [25]. Our sensitivity analysis (Text S1) estimated that in the settings of our study, the estimator is possibly biased towards the null, and the extent of the bias is modest.

\section{Conclusion}

The present MR study addressing the role of BMI in 24 traits in up to 198,502 individuals provides novel insights into the causal effect of obesity on heart failure and increased liver enzymes levels. Furthermore, to our knowledge for the first time in a well-powered sample, this study provides robust support for a causal relationship between obesity and a number of cardiometabolic traits reported previously. These results support global public prevention efforts for obesity in order to decrease costs and suffering from T2D and heart failure.

\section{Supporting Information}

Figure S1 Relationship between fixed- and randomeffects meta-analysis. (DOCX)

Figure S2 Illustration of sensitivity analysis correlation effect on the instrumental variable estimator for BMI association with ever heart failure.

(DOCX)

Table S1 Phenotypic details of the participating cohorts.

(DOCX)

Table S2 Cohort-specific genotyping details. (DOCX)

Table S3 List of proxies. (DOCX)

Table S4 Definitions of outcomes and trait transformations.

(DOCX)

Table S5 Specifications of assays used for quantitative traits and study-specific definitions of binary traits. (DOCX)

Table S6 Summary of sensitivity analysis for the effect of correlation on instrumental variable estimates. (DOCX)

Table S7 Summary of sensitivity analysis for the effect of correlation on the estimates of differences between instrumental variable and observational analysis. (DOCX)

\section{Text S1 Extended methods.}

(DOCX)

\section{Acknowledgments}

The authors would like to thank the staff in the Genetic Epidemiology Unit, Queensland Institute of Medical Research; participants in deCODE Genetics genetic studies; participants in the Malmö area cohorts (Diabetes Genetics Initiative, Malmö Prevention Project, Prevalence Prediction and Prevention of Diabetes, Malmö Diet and Cancer - cardiovascular cohort); Professor Paula Rantakallio (launch of the Northern Finland Birth Cohort 1966 and 1986 studies and initial data collection), Ms. Sarianna Vaara (data collection), Ms. Tuula Ylitalo (administration), Mr. Markku Koiranen (data management), Ms. Outi Tornwall, and Ms. Minttu Jussila (DNA biobanking) (Northern Finland Birth Cohort studies); Pascal Arp, Mila Jhamai, Marijn Verkerk, Lizbeth Herrera, and Marjolein Peters for their help in creating the Northern Finland Birth Cohort genome-wide association studies database; Karol Estrada and Maksim V. Struchalin (for their support in the creation and analysis of imputed data), study participants, the staff from the Rotterdam Study, and the participating general practitioners and pharmacists (Rotterdam Study); patients and their relatives, general practitioners, and neurologists for their contributions and P. Veraart for her help in genealogy, Jeannette Vergeer for the supervision of the laboratory work, and P. Snijders for his help in data collection (Erasmus Rucphen Family); Tomas Axelsson, Ann-Christine Wiman, and Caisa Pöntinen for their excellent assistance with genotyping (Prospective Investigation of the Vasculature in Uppsala Seniors; Swedish Twin Registry: Gender, Octo, Satsa, Harmony; Uppsala Longitudinal Study of Adult Men); participants from the Netherlands Twin Register; and Mr. V. Soo and other personnel (Estonian Genome Center of the University of Tartu).

\section{Author Contributions}

Conceived and designed the experiments: NLP MIM EI IP. Analyzed the data: Meta-analyses: TF SH RM API KF. Cohort-specific analyses: MH (DIL, WTCCCContr) APS (FTC, FINTWIN, FR, H2000) GT (deCODE) CLa (DGI, MPP, PPP) MKal (EGGUT) MKu (RS) HHMD (NTR) JSR (KORA) NRvZ (GODARTS) VH (NFBC) MM (TwinsUK) ES (MDCGV) BB (QIMR-AUSTWIN) CPN (GRAPHIC, WTCCCCase) NVR (ERF) KKr (MORGAM) TF (PIVUS, GOSH, TWINGENE, ULSAM) ASHav (FR, H2000) ADe (RS) LAD (GODARTS) MKaa (NFBC) MLN (MORGAM) NR (DIL) RDEB (RS, ERF) AMI (RS, ERF). Statistical insights: APl KF. Contributed reagents/materials/analysis tools: Administrative, technical, or material support: PA DA AJB DIB PB PSB GDS EJCD ASFD ADö PE TE AE MMF JF KF OHF CG SG LG ASHal CJH ALH ACH KH AH KHH HH JJH EH TI EI BI AJ MRJ MKal JKe FK JKe PK WK KKu TL JL LL CLi VL EL PAFM NGM MIM AM GWM ADM SM BO MOM GNAP NLP BWJHP MP APe APo CP IP WR NWR SR FR AR VSa NJS MS HS EJGS KSS JHS TDS KS VSt ACS AT UT MDT DAT TMT AGU CMvD EV JBW HW SMW GW JV JW. Wrote the first draft of the manuscript: TF SH RM APl KF NLP MIM EI IP. Contributed to the writing of the manuscript: TF SH RM API KF MH APS GT CLa MKal MKu HHMD JSR NRvZ VH MM ES BB CPN NVR KKr HS ASHav ADe LAD MKaa MLN NR RDEB AMI NA AJB PSB ASFD ADö PE TE OHF SG ALH KH KHH HH JJH EH TI AI BI LK JKe WK KKu TL JL CLi VL EL NWR SM APo WR FR AR MS EJGS KSS JHS VSt ACS AT MDT AGU SMW GW JW MP AE JF JV FK DAT DA PA MMF PB ASHal ACH PAFM NGM GWM JBW AJ PK BO CMvD BWJHP GDS JKe NJS CG APe HW DIB EJCD TMT CP CJH TDS LL MOM CNAP ADM LG MRJ VSa EV AH SR AM UT KS NLP MIM EI IP. ICMJE criteria for authorship read and met: TF SH RM APl KF MH APS GT CLa MKal MKu HHMD JSR NRvZ VH MM ES BB CPN NVR KKr HS ASHav ADe LAD MKaa MLN NR RDEB AMI NA AJB PSB ASFD ADö PE TE OHF SG ALH KH KHH HH JJH EH TI AI BI LK JKe WK KKu TL JL CLi VL EL NWR SM APo WR FR AR MS EJGS KSS JHS VSt ACS AT MDT AGU SMW GW JW MP AE JF JV FK DAT DA PA MMF PB ASHal ACH PAFM NGM GWM JBW AJ PK BO CMvD BWJHP GDS JKe NJS CG APe HW DIB EJCD TMT CP CJH TDS LL MOM CNAP ADM LG MRJ VSa EV AH SR AM UT KS NLP MIM EI IP. Agree with manuscript results and conclusions: TF SH RM API KF MH APS GT CLa MKal MKu HHMD JSR NRvZ VH MM ES BB CPN NVR KKr HS ASHav ADe LAD MKaa MLN NR RDEB AMI NA AJB PSB ASFD ADö PE TE OHF SG ALH KH KHH HH JJH EH TI AI BI LK JKe WK KKu TL JL CLi VL EL NWR SM APo WR FR AR MS EJGS KSS JHS VSt ACS AT MDT AGU SMW GW JW MP AE JF JV FK DAT DA PA MMF PB ASHal ACH PAFM NGM GWM JBW AJ PK BO GMvD BWJHP GDS JKe NJS CG APe HW DIB EJCD TMT CP GJH TDS LL MOM CNAP ADM LG MRJ VSa EV AH SR AM UT KS NLP MIM EI IP. Enrolled patients: Acquisition of data: PA DA AJB DIB PB PSB GDS EJCD ASFD ADö PE TE AE MMF JF KF OHF CG SG LG ASHal CJH ALH ACH KH AH KHH HH JJH EH TI EI BI AJ MRJ MKalJKe FK JKe PK WK KKu TL JL LL CLi VL EL PAFM NGM MIM AM GWM ADM SM BO MOM GNAP NLP BWJHP MP APe APo CP IP WR NWR SR FR AR VSa NJS MS EJGS KSS JHS TDS KS VSt ACS AT UT MDT DAT TMT AGU GMvD EV JBW HW SMW GW JV JW. Obtained funding: PA DA AJB DIB PB PSB GDS EJCD ASFD ADö PE TE AE MMF JF KF OHF CG SG LG ASHal CJH ALH ACH KH AH KHH HH JJH EH TI EI BI AJ MRJ MKal JKe FK JKe PK WK KKu TL JL LL GLi VL EL PAFM 
NGM MIM AM GWM ADM SM BO MOM CNAP NLP BWJHP MP APe APo CP IP WR NWR SR FR AR VSa NJS MS EJGS KSS JHS TDS KS VSt ACS AT UT MDT DAT TMT AGU GMvD EV JBW HW SMW GW JV JW. Statistical analysis: TF SH RM API KF NLP MIM EI IP MH

\section{References}

1. Gersh BJ, Sliwa K, Mayosi BM, Yusuf S (2010) Novel therapeutic concepts: the epidemic of cardiovascular disease in the developing world: global implications. Eur Heart J 31: 642-648.

2. Whitlock G, Lewington S, Sherliker P, Clarke R, Emberson J, et al. (2009) Bodymass index and cause-specific mortality in 900000 adults: collaborative analyses of 57 prospective studies. Lancet 373: 1083-1096.

3. Davey Smith G, Sterne JA, Fraser A, Tynelius P, Lawlor DA, et al. (2009) The association between BMI and mortality using offspring BMI as an indicator of own BMI: large intergenerational mortality study. BMJ 339: b5043.

4. Knowler WC, Barrett-Connor E, Fowler SE, Hamman RF, Lachin JM, et al. (2002) Reduction in the incidence of type 2 diabetes with lifestyle intervention or metformin. N Engl J Med 346: 393-403.

5. Tuomilehto J, Lindstrom J, Eriksson JG, Valle TT, Hamalainen H, et al. (2001) Prevention of type 2 diabetes mellitus by changes in lifestyle among subjects with impaired glucose tolerance. N Engl J Med 344: 1343-1350.

6. Pan XR, Li GW, Hu YH, Wang JX, Yang WY, et al. (1997) Effects of diet and exercise in preventing NIDDM in people with impaired glucose tolerance. The Da Qing IGT and Diabetes Study. Diabetes Care 20: 537-544.

7. Li G, Zhang P, Wang J, Gregg EW, Yang W, et al. (2008) The long-term effect of lifestyle interventions to prevent diabetes in the China Da Oing Diabetes Prevention Study: a 20-year follow-up study. Lancet 371: 1783-1789.

8. Uusitupa M, Peltonen M, Lindstrom J, Aunola S, Ilanne-Parikka P, et al. (2009) Ten-year mortality and cardiovascular morbidity in the Finnish Diabetes Prevention Study - secondary analysis of the randomized trial. PLoS ONE 4: e5656. doi:10.1371/journal.pone.0005656

9. Davey Smith G, Ebrahim S (2003) 'Mendelian randomization': can genetic epidemiology contribute to understanding environmental determinants of disease? Int J Epidemiol 32: 1-22.

10. Didelez V, Sheehan N (2007) Mendelian randomization as an instrumental variable approach to causal inference. Stat Methods Med Res 16: 309-330.

11. Sheehan NA, Meng S, Didelez V (2011) Mendelian randomisation: a tool for assessing causality in observational epidemiology. In: Teare MD, editor. Genetic epidemiology. New York City: Humana Press.

12. Day FR, Loos RJ (2011) Developments in obesity genetics in the era of genomewide association studies. J Nutrigenet Nutrigenomics 4: 222-238.

13. Speliotes EK, Willer CJ, Berndt SI, Monda KL, Thorleifsson G, et al. (2010) Association analyses of 249,796 individuals reveal 18 new loci associated with body mass index. Nat Genet 42: 937-948.

14. Timpson NJ, Nordestgaard BG, Harbord RM, Zacho J, Frayling TM, et al. (2011) C-reactive protein levels and body mass index: elucidating direction of causation through reciprocal Mendelian randomization. Int J Obes (Lond) 35: 300-308.

15. Freathy RM, Timpson NJ, Lawlor DA, Pouta A, Ben-Shlomo Y, et al. (2008) Common variation in the FTO gene alters diabetes-related metabolic traits to the extent expected given its effect on BMI. Diabetes 57: 1419-1426.

16. Nordestgaard BG, Palmer TM, Benn M, Zacho J, Tybjaerg-Hansen A, et al. (2012) The effect of elevated body mass index on ischemic heart disease risk: causal estimates from a mendelian randomisation approach. PLoS Med 9: e1001212. doi:10.1371/journal.pmed. 1001212

17. Kivimaki M, Smith GD, Timpson NJ, Lawlor DA, Batty GD, et al. (2008) Lifetime body mass index and later atherosclerosis risk in young adults: examining causal links using Mendelian randomization in the Cardiovascular Risk in Young Finns study. Eur Heart J 29: 2552-2560.

18. Timpson NJ, Harbord R, Davey Smith G, Zacho J, Tybjaerg-Hansen A, et al. (2009) Does greater adiposity increase blood pressure and hypertension risk? Mendelian randomization using the FTO/MC4R genotype. Hypertension 54: 84-90.

19. Welsh P, Polisecki E, Robertson M, Jahn S, Buckley BM, et al. (2010) Unraveling the directional link between adiposity and inflammation: a bidirectional Mendelian randomization approach. J Clin Endocrinol Metab 95: 93-99.

20. Grundy SM, Cleeman JI, Daniels SR, Donato KA, Eckel RH, et al. (2005) Diagnosis and management of the metabolic syndrome: an American Heart Association/National Heart, Lung, and Blood Institute Scientific Statement. Circulation 112: 2735-2752.
APS GT CLa MKal MKu HHMD JSR NRvZ VH MM ES BB CPN NVR KKr ASHav ADe LAD MKaa MLN. Access to all of the data in the study and responsibility for the integrity of the data and the accuracy of the data analysis: TF SH RM API KF NLP MIM EI IP.

21. Cox D (1972) Regression models and life-tables (with discussion). J R Stat Soc Series B Stat Methodol 34: 187-220.

22. Ross I, Robert G (1996) R: A language for data analysis and graphics. J Comput Graph Stat 5: 299-314.

23. Magi R, Morris AP (2010) GWAMA: software for genome-wide association meta-analysis. BMC Bioinformatics 11: 288

24. Wald A (1940) The fitting of straight lines if both variables are subject to error. Ann Math Stat 11: 284-300.

25. Palmer TM, Sterne JA, Harbord RM, Lawlor DA, Sheehan NA, et al. (2011) Instrumental variable estimation of causal risk ratios and causal odds ratios in Mendelian randomization analyses. Am J Epidemiol 173: 1392-1403.

26. Li H, Kilpelainen TO, Liu C, Zhu J, Liu Y, et al. (2012) Association of genetic variation in FTO with risk of obesity and type 2 diabetes with data from 96,551 East and South Asians. Diabetologia 55: 981-995.

27. Meyre D (2012) Is FTO a type 2 diabetes susceptibility gene? Diabetologia 55: 873-876.

28. Kenchaiah S, Evans JC, Levy D, Wilson PW, Benjamin EJ, et al. (2002) Obesity and the risk of heart failure. N Engl J Med 347: 305-313

29. Ingelsson E, Sundstrom J, Arnlov J, Zethelius B, Lind L (2005) Insulin resistance and risk of congestive heart failure. JAMA 294: 334-341.

30. Wilhelmsen L, Rosengren A, Eriksson H, Lappas G (2001) Heart failure in the general population of men - morbidity, risk factors and prognosis. J Intern Med 249: 253-261.

31. Manson JE, Colditz GA, Stampfer MJ, Willett WC, Rosner B, et al. (1990) A prospective study of obesity and risk of coronary heart disease in women. N Engl J Med 322: 882-889.

32. Lauer MS, Anderson KM, Kannel WB, Levy D (1991) The impact of obesity on left ventricular mass and geometry. The Framingham Heart Study. JAMA 266: 231-236.

33. Vincent HK, Powers SK, Stewart DJ, Shanely RA, Demirel H, et al. (1999 Obesity is associated with increased myocardial oxidative stress. Int J Obes Relat Metab Disord 23: 67-74

34. Zhou YT, Grayburn P, Karim A, Shimabukuro M, Higa M, et al. (2000) Lipotoxic heart disease in obese rats: implications for human obesity. Proc Natl Acad Sci U S A 97: 1784-1789.

35. World Health Organization (2008) The global burden of disease: 2004 update. Geneva: World Health Organization.

36. Roger VL, Go AS, Lloyd-Jones DM, Adams RJ, Berry JD, et al. (2011) Heart disease and stroke statistics - 2011 update: a report from the American Heart Association. Circulation 123: e18-e209.

37. Fu S, Yang L, Li P, Hofmann O, Dicker L, et al, (2011) Aberrant lipid metabolism disrupts calcium homeostasis causing liver endoplasmic reticulum stress in obesity. Nature 473: 528-531

38. Sheehan NA, Didelez V, Burton PR, Tobin MD (2008) Mendelian randomisation and causal inference in observational epidemiology. PLoS Med 5: e177. doi:10.1371/journal.pmed.0050177

39. Smith GD, Lawlor DA, Harbord R, Timpson N, Day I, et al. (2007) Clustered environments and randomized genes: a fundamental distinction between conventional and genetic epidemiology. PLoS Med 4: e352. doi:10.1371/ journal.pmed.0040352

40. Church C, Moir L, McMurray F, Girard C, Banks GT, et al. (2010) Overexpression of Fto leads to increased food intake and results in obesity. Nat Genet 42: 1086-1092.

41. Cecil JE, Tavendale R, Watt P, Hetherington MM, Palmer CN (2008) An obesity-associated FTO gene variant and increased energy intake in children. N Engl J Med 359: 2558-2566.

42. Palmer TM, Lawlor DA, Harbord RM, Sheehan NA, Tobias JH, et al. (2011) Using multiple genetic variants as instrumental variables for modifiable risk factors. Stat Methods Med Res 21: 223-242.

43. Willer CJ, Speliotes EK, Loos RJ, Li S, Lindgren CM, et al. (2009) Six new loci associated with body mass index highlight a neuronal influence on body weight regulation. Nat Genet 41: 25-34.

44. Frayling TM, Timpson NJ, Weedon MN, Zegoini E, Freathy RM, et al. (2007) A common variant in the FTO gene is associated with body mass index and predisposes to childhood and adult obesity. Science 316: 889-894. 


\section{Editors' Summary}

Cardiovascular disease (CVD)—disease that affects the heart and/or the blood vessels-is a major cause of illness and death worldwide. In the US, for example, coronary heart disease-a CVD in which narrowing of the heart's blood vessels by fatty deposits slows the blood supply to the heart and may eventually cause a heart attack-is the leading cause of death, and stroke-a CVD in which the brain's blood supply is interrupted-is the fourth leading cause of death. Globally, both the incidence of CVD (the number of new cases in a population every year) and its prevalence (the proportion of the population with CVD) are increasing, particularly in low- and middle-income countries. This increasing burden of CVD is occurring in parallel with a global increase in the incidence and prevalence of obesityhaving an unhealthy amount of body fat (adiposity) - and of metabolic diseases-conditions such as diabetes in which metabolism (the processes that the body uses to make energy from food) is disrupted, with resulting high blood sugar and damage to the blood vessels.

Why Was This Study Done? Epidemiological studiesinvestigations that record the patterns and causes of disease in populations-have reported an association between adiposity (indicated by an increased body mass index [BMI], which is calculated by dividing body weight in kilograms by height in meters squared) and cardiometabolic traits such as coronary heart disease, stroke, heart failure (a condition in which the heart is incapable of pumping sufficient amounts of blood around the body), diabetes, high blood pressure (hypertension), and high blood cholesterol (dyslipidemia). However, observational studies cannot prove that adiposity causes any particular cardiometabolic trait because overweight individuals may share other characteristics (confounding factors) that are the real causes of both obesity and the cardiometabolic disease. Moreover, it is possible that having CVD or a metabolic disease causes obesity (reverse causation). For example, individuals with heart failure cannot do much exercise, so heart failure may cause obesity rather than vice versa. Here, the researchers use "Mendelian randomization" to examine whether adiposity is causally related to various cardiometabolic traits. Because gene variants are inherited randomly, they are not prone to confounding and are free from reverse causation. It is known that a genetic variant (rs9939609) within the genome region that encodes the fat-mass- and obesityassociated gene (FTO) is associated with increased BMI. Thus, an investigation of the associations between rs9939609 and cardiometabolic traits can indicate whether obesity is causally related to these traits.

What Did the Researchers Do and Find? The researchers analyzed the association between rs9939609 (the "instrumental variable," or IV) and BMI, between rs9939609 and 24 cardiometabolic traits, and between BMI and the same traits using genetic and health data collected in 36 populationbased studies of nearly 200,000 individuals of European descent. They then quantified the strength of the causal association between BMI and the cardiometabolic traits by calculating "IV estimators." Higher BMI showed a causal relationship with heart failure, metabolic syndrome (a combination of medical disorders that increases the risk of developing (VD), type 2 diabetes, dyslipidemia, hypertension, increased blood levels of liver enzymes (an indicator of liver damage; some metabolic disorders involve liver damage), and several other cardiometabolic traits. All the IV estimators were similar to the BMI-cardiovascular trait associations (observational estimates) derived from the same individuals, with the exception of diabetes, where the causal estimate was higher than the observational estimate, probably because the observational estimate is based on a single BMI measurement, whereas the causal estimate considers lifetime changes in BMI.

What Do These Findings Mean? Like all Mendelian randomization studies, the reliability of the causal associations reported here depends on several assumptions made by the researchers. Nevertheless, these findings provide support for many previously suspected and biologically plausible causal relationships, such as that between adiposity and hypertension. They also provide new insights into the causal effect of obesity on liver enzyme levels and on heart failure. In the latter case, these findings suggest that a oneunit increase in BMI might increase the incidence of heart failure by $17 \%$. In the US, this corresponds to 113,000 additional cases of heart failure for every unit increase in BMI at the population level. Although additional studies are needed to confirm and extend these findings, these results suggest that global efforts to reduce the burden of obesity will likely also reduce the occurrence of CVD and metabolic disorders.

Additional Information. Please access these websites via the online version of this summary at http://dx.doi.org/10. 1371/journal.pmed.1001474.

- The American Heart Association provides information on all aspects of cardiovascular disease and tips on keeping the heart healthy, including weight management (in several languages); its website includes personal stories about stroke and heart attacks

- The US Centers for Disease Control and Prevention has information on heart disease, stroke, and all aspects of overweight and obesity (in English and Spanish)

- The UK National Health Service Choices website provides information about cardiovascular disease and obesity, including a personal story about losing weight

- The World Health Organization provides information on obesity (in several languages)

- The International Obesity Taskforce provides information about the global obesity epidemic

- Wikipedia has a page on Mendelian randomization (note: Wikipedia is a free online encyclopedia that anyone can edit; available in several languages)

- MedlinePlus provides links to other sources of information on heart disease, on vascular disease, on obesity, and on metabolic disorders (in English and Spanish)

- The International Association for the Study of Obesity provides maps and information about obesity worldwide

- The International Diabetes Federation has a web page that describes types, complications, and risk factors of diabetes 